\title{
Spectrum Allocation Strategies for Heterogeneous Networks
}

\author{
Rindra Ramamonjison ${ }^{1}$, Gia Khanh $\operatorname{Tran}^{1}$, Kei Sakaguchi ${ }^{1}$, Kiyomichi Araki ${ }^{1}$, Shoji Kaneko ${ }^{2}$, Yoji $\mathrm{Kishi}^{2}$, Noriaki Miyazaki ${ }^{2}$ \\ ${ }^{1}$ Tokyo Institute of Technology, Japan \\ ${ }^{2}$ KDDI R\&D Laboratories Inc, Japan
}

\begin{abstract}
This work analyzed the trade-off between the area spectral efficiency and outage user rates in heterogeneous cellular networks with overlapping picocells. We considered two different models for the spectrum allocation and cell association. The first model employs a spectrum overlapping strategy with an SINR-based cell association. The second model avoids the interference between macrocell and picocell through a spectrum splitting strategy. Furthermore, picocell range expansion was also considered for the later to enable a load balancing between the macrocell and picocells. Our results showed that while the spectrum overlapping provides the highest spectral efficiency, its outage rate performance is very poor. In contrast, the spectrum splitting offered a trade-off by guaranteeing a minimum QoS for the weak user rates and by flexibly varying the spectrum splitting ratio and picocell range.
\end{abstract}

\section{INTRODUCTION}

With the increasing popularity of mobile internet services, the scarcity of the spectrum poses formidable challenges for wireless network operators. To support the heavy user demand, the operators continually deploy more cells and reuse the spectrum in distant cells. In fact, getting the users closer to the serving base station significantly improves the coverage and the user throughput. However, dense cellular deployment is very expensive in macrocellular networks. Therefore, deploying cheap access points at homes or in public hotspots seems to be a better approach [1]. Due to the difference of the macrocell BS and the new picocell or femtocell access points, the cellular network becomes heterogeneous.

Heterogeneous networks extend the hierarchical cell structure (HCS) of more traditional macrocell-microcell deployments [2], [3]. Early works in these networks have studied the frequency planning by considering the capacity, hand-off and mobility of the users [2], [4]. In contrast to macrocellmicrocell deployment, future heterogeneous networks will consist of a dense deployment of picocell and femtocell base stations, whose coverages overlap with the macrocell's. Consequently, the major concerns are the spectrum efficiency and interference in heterogeneous networks [1], [5]. The main objective of introducing heterogeneous cells is to improve both the spectral efficiency and quality of service across the whole network service area.

Recently, femtocell networks have attracted a lot of attention (c.f. [6], [7]). However, they differ from picocells by their smaller coverage and transmit power as they are targeted mainly for home users [1]. In addition, the femtocell BSs are deployed by users so that their locations are random. Therefore, research on femtocell networks focus on the dynamic resource allocation and interference in both closed and open access networks.

In contrast, we consider the macrocell-picocell heterogeneous networks and investigate two general spectrum allocation models: spectrum overlapping and spectrum splitting. In the former, the spectrum is fully reused and an SINR-based rule is employed for cell association. In the later, orthogonal bandwidths are allocated to the macrocell and picocells to avoid the interference. Also, we introduce the picocell range expansion in the spectrum splitting model to enable a flexible load balancing between the macrocell and picocells.

Dynamic resource allocation and cell association schemes have been proposed in a recent work for picocells [10]. However, it did not fully analyze the spectrum allocation problem on a macroscale level. In fact, a better understanding of the trade-offs associated with different spectrum management strategies is important for heterogeneous cell planning and is also useful for designing and evaluating the benefits of intercell coordination schemes.

Since a heterogeneous deployment should improve both the efficiency and quality of service accross the cells, our aim is to bring insights into the area spectral efficiency/outage rate trade-offs associated with the spectrum overlapping and spectrum splitting models. The paper is organized as follows. In Section II, we introduce the model of the heterogeneous networks. Sections III and IV present the spectrum overlapping and spectrum splitting strategies with their corresponding performance metrics and design parameters. In Section V, we show the analysis on the trade-off between spectral efficiency and outage rates through simulations.

\section{HeTERogeneous NETWORK MODELS}

We consider a heterogeneous network with $N_{p}$ picocells overlapping the coverage of a large macrocell with radius $R_{M}$ and area $S=\pi R_{M}^{2}$. The picocell BSs are deployed at a distance $D$ from the macro BS. An example of an heterogeneous deployment is shown in Figure 1. We denote by $P_{M}$ and $P_{p}$ the transmit powers of the macro BS and the picocell BSs respectively. Although a dynamic power allocation across the subchannels of users is expected to bring further gain, we assume in this study that each base station transmits to its served users with equal power. 
For simplicity, we assume that the users are uniformly distributed across the network coverage and that the cells are fully loaded. In addition, each cell served by its users using a Round Robin scheduling.

\section{A. Cell association rules}

Heterogeneous networks are flexible since the users have a diverse choice of serving cells with different transmit parameters and resources. In fact, various network factors such as quality of service, load-balancing, or spectrum efficiency can be managed depending on the cell association rule. In this work, we consider two mechanisms for cell association:

- cell range expansion, i.e. a user is associated to the closest picocell if that user is within a specified maximum range $R_{p}$. This cell association rule can reduce the load of the macrocell users by increasing the range $R_{p}$.

- SINR-based cell association, i.e. a user is assigned to the macrocell or a picocell which provides the highest SINR to that user.

\section{B. Spectrum allocation and area spectral efficiency}

In this work, we study two general strategies for managing the spectrum in heterogeneous networks:

- spectrum splitting strategy (1) allocates orthogonal bandwidths for the macrocell and the picocells. Let $W$ denote the available spectrum bandwidth. Then, a splitting ratio $0 \leq \rho \leq 1$ controls the allocation such that $W_{M}=\rho W$ is allocated to the macrocell and $W_{p}=(1-\rho) W$ is used by all picocells. In this case, there is no interference between the macrocell and a picocell. Interference only occurs between picocells but the effect is limited when the picocell BSs' transmit power is low or when they are deployed by operators with enough separation.

- spectrum overlapping strategy (2) allows the macrocell and the picocells to fully reuse the spectrum at the cost of interference, i.e. $W_{M}=W_{p}=W$.

For each of these strategies, the performance metric of interest is the average area spectral efficiency (ASE), defined as the sum of the maximum average throughput per unit bandwidth per unit area that the heterogeneous network can support[11]:

$$
A S E=\frac{W_{M} \bar{C}_{M}+W_{p} \sum_{p=1}^{N_{p}} \bar{C}_{p}}{S \times W} \quad\left[\mathrm{bps} / \mathrm{Hz} / \mathrm{m}^{2}\right]
$$

where $\bar{C}_{M}=\mathbb{E}_{S_{M}}\left[C_{M}\right]$ and $\bar{C}_{p}=\mathbb{E}_{S_{p}}\left[C_{p}\right]$ are the cell throughput per $\mathrm{Hz}$ averaged over the user's location in the macrocell or in the picocell's total service areas $S_{M}, S_{p}$ respectively.

In this study, we assumed that adaptive modulation and coding (AMC) scheme is employed to improve the throughput based on the channel conditions. To take into account the AMC scheme and the losses due to implementation issues, the following modified Shannon capacity formula was used to calculate the maximum achievable throughput [12], [13]:

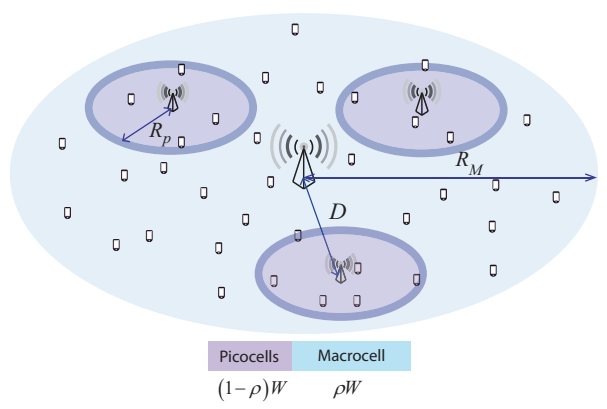

Figure 1. Heterogeneous network with a spectrum splitting model

$$
C=\left\{\begin{array}{cc}
\eta_{W} \log _{2}\left(1+\eta_{S I N R} \gamma\right), & \gamma \leq S I N R_{\max } \\
C_{\max } & \gamma>S I N R_{\max }
\end{array}\right.
$$

where $\gamma$ is the SINR; and the bandwidth efficiency $\eta_{W}$ and SINR efficiency $\eta_{S I N R}$ are obtained through curving fitting of the throughputs achievable with different modualtion and code rates of the AMC scheme.

\section{SPECTRUM OVERLAPPING MODEL}

Since the spectrum is fully reused across the heterogeneous network, each user receives interference from all the other cells. Thus, it is reasonable to use the SINR-based cell association rule for this model. In practice, this rule can be implemented during cell search.

Deriving analytical expressions of the average cell throughputs $\bar{C}_{1, M}, \bar{C}_{1, p}$ are computationally burdensome since it requires integrations of (2) over non-circular service areas $S_{1, M}, S_{1, p}$. Here, the index 1 denotes the parameters associated with the spectrum overlapping model. Therefore, it is common to approximate $\bar{C}_{1, M}, \bar{C}_{1, p}$ as well as the ASE through simulations [11]. In the spectrum overlapping model, the ASE is a function of the picocell transmit power $P_{p}$ as this later determines the macrocell and picocell service areas:

$$
A S E_{1}\left(P_{p}\right)=\frac{\bar{C}_{1, M}\left(P_{p}\right)+\sum_{k=1}^{N_{p}} \bar{C}_{1, p, k}\left(P_{p}\right)}{S}
$$

Subsequently, we define the macrocell and picocells' average user throughput by:

$$
\begin{aligned}
\bar{T}_{1, M}\left(P_{p}\right) & =\frac{\bar{C}_{1, M}\left(P_{p}\right)}{S_{1, M}\left(P_{p}\right)} \\
\bar{T}_{1, p}\left(P_{p}\right) & =\frac{\sum_{k=1}^{N_{p}} \bar{C}_{1, p, k}\left(P_{p}\right)}{S_{1, p}\left(P_{p}\right)},
\end{aligned}
$$

where $\bar{C}_{1, p, k}$ denotes the average cell throughput of the $k$-th picocell for the overlapping model.

Note that instead of dividing the cell throughput by the number of served users, we calculate the statistical averages of the macrocell and picocell user rates using the fact that the user distribution is uniform. Therefore, we divide the cell throughput by the service areas, as in (4) and (5), so that the statistical average user rates are independent of the actual number of users in the system and are expressed with the same unit $\mathrm{bps} / \mathrm{Hz} / \mathrm{m}^{2}$ as the ASE. 


\section{SPECTRUM SPLITTING MODEL}

To avoid the interference between the macrocell and the picocells, the spectrum splitting model allocates orthogonal subbands to these cells. Furthermore, the cell range expansion is implemented so that a user is assigned to its closest picocell BS if it is within the picocell BS's range $R_{p}$. Similarly to the previous model, we use the index 2 to refer to the parameters of the spectrum splitting model.

\section{A. Area spectral efficiency}

The average ASE is a function of the splitting ratio and picocell range:

$$
A S E_{2}\left(\rho, R_{p}\right)=\frac{\rho \bar{C}_{2, M}\left(R_{p}\right)+(1-\rho) \sum_{k=1}^{N_{p}} \bar{C}_{2, p, k}\left(R_{p}\right)}{S}
$$

where $\bar{C}_{2, M}$ and $\bar{C}_{2, p}$ are the cell throughput averaged over the user's location in the service areas $S_{2, M}, S_{2, p}$ respectively. In contrast to the spectrum overlapping model, each picocell's service area is circular. Furthermore, the average user throughputs or spectral efficiency $\bar{T}_{2, M}, \bar{T}_{2, p}$ in the macrocell and picocells are:

$$
\begin{aligned}
\bar{T}_{2, M}\left(\rho, R_{p}\right) & =\frac{\rho \times \bar{C}_{2, M}\left(R_{p}\right)}{S_{2, M}\left(R_{p}\right)} \\
\bar{T}_{2, p}\left(\rho, R_{p}\right) & =\frac{(1-\rho) \times \sum_{k=1}^{N_{p}} \bar{C}_{2, p, k}\left(R_{p}\right)}{S_{2, p}\left(R_{p}\right)}
\end{aligned}
$$

\section{B. Optimal splitting ratio and picocell range}

In the spectrum splitting model, the spectrum allocation ratio and picocell range $\left(\rho^{\star}, R_{p}^{\star}\right)$ are design parameters that could be used to maximize the ASE 6. However, if no constraint is imposed on the quality of service (QoS) of the users (precisely the average user rates $\bar{T}_{2, M}$ and $\bar{T}_{2, p}$ ), the optimal choice simply reduces to $\rho^{\star}=0$ or $\rho^{\star}=1$ since the ASE 6 is a convex combination of $\bar{C}_{2, M}\left(R_{p}\right)$ and $\sum_{k=1}^{N_{p}} \bar{C}_{2, p, k}\left(R_{p}\right)$. If $\sum_{p=1}^{N_{p}} \bar{C}_{1, p}>\bar{C}_{1, M}$ for example, i.e. the aggregate cell throughput of picocells is larger than the macrocell, then allocating the whole spectrum to the picocells, i.e. $\rho^{\star}=0$, maximizes the spectral efficiency. Conversely, the macrocell users would receive the whole spectrum whereas picocell users starve if $\sum_{p=1}^{N_{p}} \bar{C}_{1, p}<\bar{C}_{1, M}$.

Therefore, it becomes important to enforce some fairness constraints as follows:

$$
\begin{aligned}
& \underset{\rho, R_{p}}{\operatorname{maximize}} A S E_{2} \\
& \text { subject to } \min \left(\bar{T}_{1, p}, \bar{T}_{1, M}\right) \geq \bar{\Gamma}_{\text {min }}
\end{aligned}
$$

where $\bar{\Gamma}_{\min }$ is the minimum average user throughput.

To find the optimal pair $\left(\rho^{\star}, R_{p}^{\star}\right)$, we use the fact that the constraint (10) should be active at an optimal point. Therefore, if $\sum_{p=1}^{N_{p}} \bar{C}_{2, p}\left(R_{p}\right)>\bar{C}_{2, M}\left(R_{p}\right)$ for given picocell range $R_{p}$ and transmit power $P_{p}$, then we find the minimum

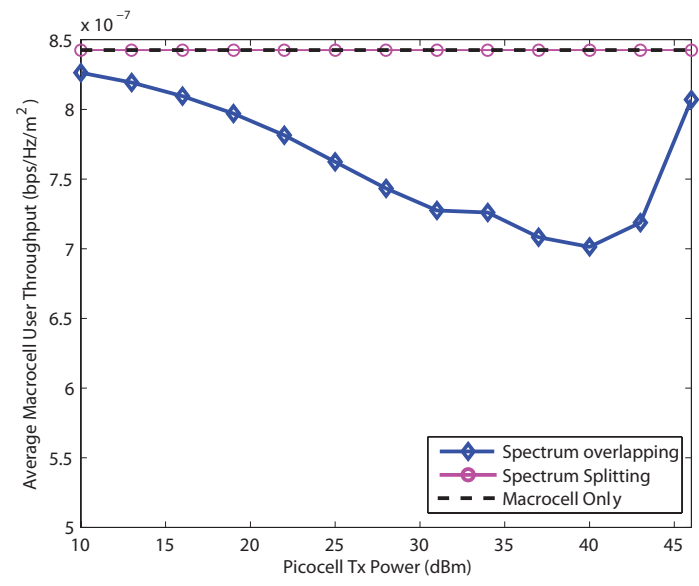

Figure 2. Average Macrocell User Rates vs. Picocell Tx Power

$\rho^{\star}=\rho_{\min }$ which maximizes the ASE while satisfying (10) (i.e. $\bar{T}_{2, M}(\rho)=\bar{\Gamma}_{\text {min }}$ ) as follows:

$$
\rho_{\min }\left(R_{p}\right)=\frac{S_{2, M}\left(R_{p}\right) \cdot \bar{\Gamma}_{\min }}{\bar{C}_{2, M}\left(R_{p}\right)}
$$

In the case that $\sum_{p=1}^{N_{p}} \bar{C}_{2, p}\left(R_{p}\right) \leq \bar{C}_{2, M}\left(R_{p}\right)$, the optimal ratio $\rho^{\star}=\rho_{\max }$ which maximizes the ASE while satisfying (10) (i.e. $\left.\bar{T}_{2, p}(\rho)=\bar{\Gamma}_{\text {min }}\right)$ is similarly given by:

$$
\rho_{\max }\left(R_{p}\right)=1-\frac{S_{2, p}\left(R_{p}\right) \cdot \bar{\Gamma}_{\text {min }}}{\sum_{p=1}^{N_{p}} \bar{C}_{2, p}}
$$

Consequently, we can search for the jointly optimal $\left(\rho^{\star}, R_{p}^{\star}\right)$ numerically using (11) and (12). Precisely, we vary the range of the picocell range in the simulation; then for a given $R_{p}$, we compute the corresponding optimal $\rho^{\star}\left(R_{p}\right)$ and corrresponding spectral efficiency $A S E_{2}\left(\rho^{\star}\left(R_{p}\right)\right)$. Then, the optimal picocell range is taken as:

$$
\begin{gathered}
R_{p}^{\star}=\underset{R_{p}}{\operatorname{argmax}} A S E_{2}\left(\rho^{\star}\left(R_{p}\right)\right) \\
\text { V. NUMERICAL ANALYSIS }
\end{gathered}
$$

\section{NUMERICAL ANALYSIS}

In this section, we present the numerical results for a heterogeneous network with one macrocell and $N_{p}=4$ picocells. In the simulation, we average the spectral efficiency by generating a large number of uniformly distributed user locations and apply the cell association rules in Section II. The simulation parameters are summarized in Table I. The picocell transmit power was varied between $10 \mathrm{dBm}$ and $46 \mathrm{dBm}$ which is the transmit power of the macrocell BS. For the spectrum splitting model, the QoS parameter $\bar{\Gamma}_{\text {min }}$, i.e. the minimum average user rate constraint, was set equal to the achieved average user rate of a macrocell-only case. Using this constraint as a reference for comparison, we see in Figure 2 that the average macrocell user rates for the spectrum overlapping did not satisfy the constraint whereas the splitting model did.

Figure 3 highlights the trade-off between the area spectral efficiency and outage user rates for the spectrum allocation 


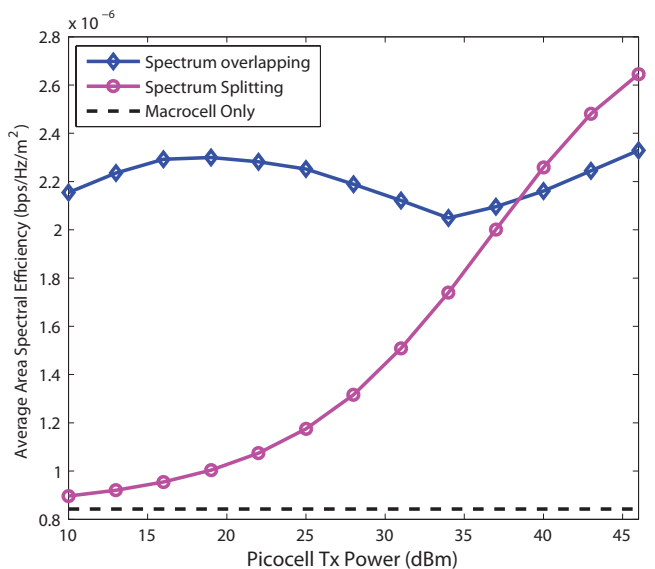

(a) Average Area Spectral Efficiency

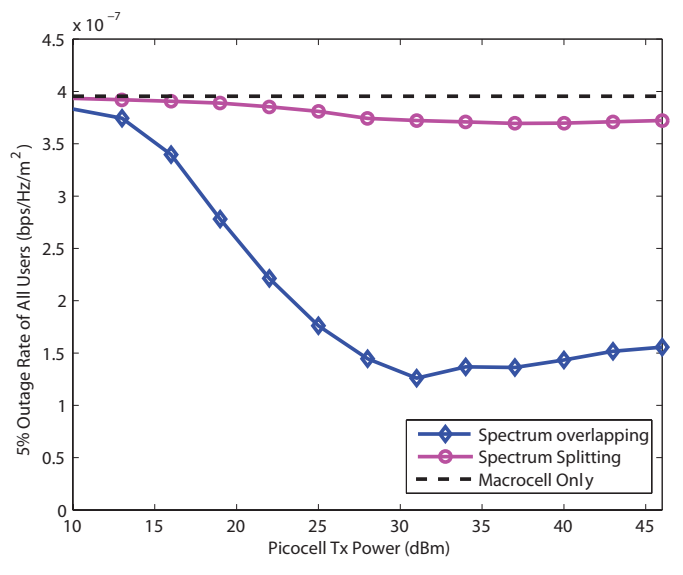

(b) Outage Rate of all users

Figure 3. (a) Area Spectral Efficiency vs. (b) Outage Rate performance of users

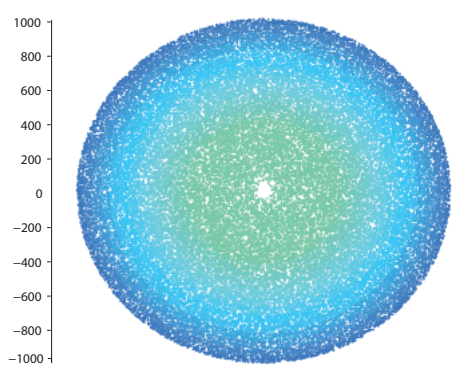

(a)

No Picocells

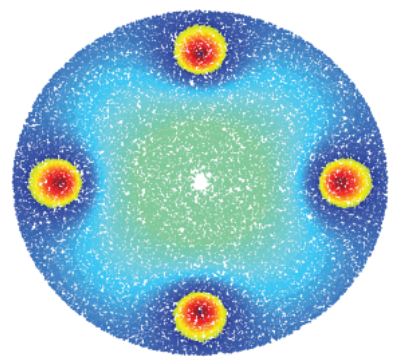

(b)

Spectrum Overlapping Pico Tx power $=28 \mathrm{dBm}$

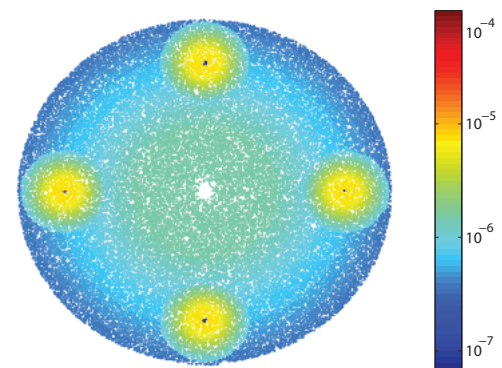

(c)

Spectrum Splitting $\left(\rho^{*}, R_{p}^{*}\right)=(0.72,240 \mathrm{~m})$ Pico Tx power $=28 \mathrm{dBm}$

Figure 4. Average User Throughputs with (a) No Picocells, (b) Heterogeneous cells with spectrum overlapping, and (c) Heterogeneous cells with spectrum splitting.

strategies discussed in this paper. We clearly see that, for a reasonable range of picocell transmit power, the spectrum overlapping model achieved higher spectrum efficiency than the spectrum splitting model. In addition, both models of spectrum allocation could improve the spectral efficiency compared to the macrocell-only scenario, showing the advantage of heterogeneous networks.

However, when we look at the 5\% outage rate of all users, the performance of the spectrum overlapping model significantly degraded as the picocell transmit power increases. This degradation was especially due to the severe interference created to the macrocell users near the picocell BS (see Figure 4). In fact, Figure 6 confirms that the users in outage were clearly the macrocell users. In contrast to the spectrum overlapping model, the spectrum splitting allocation could improve the spectral efficiency while guaranteeing the QoS constraint for the weaker users. Although the spectral efficiency gain is modest, the spectrum splitting model offered a better flexibility by optimally controlling the spectrum splitting ratio and picocell range.

These optimal parameters are shown in Figure 5. It is understood that the optimal spectrum splitting ratio $\rho^{\star}$ was close to 1 when the picocell transmit power is low, i.e. most

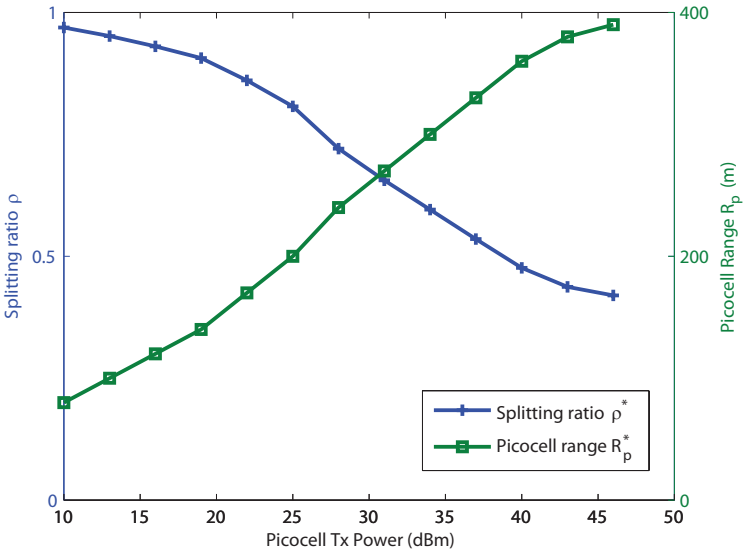

Figure 5. Optimal spectrum splitting ratio $\rho^{\star}$ and picocell range $R_{p}^{\star}$

of the spectrum is allocated to the macrocell. In that case, most users were assigned to the macrocell BS so that a large portion of the spectrum is needed to serve these users. With increasing picocell transmit power, the splitting ratio gradually decreased whereas the optimal picocell range $R_{p}^{\star}$ increased. Although not included in this paper, our results showed, however, that 

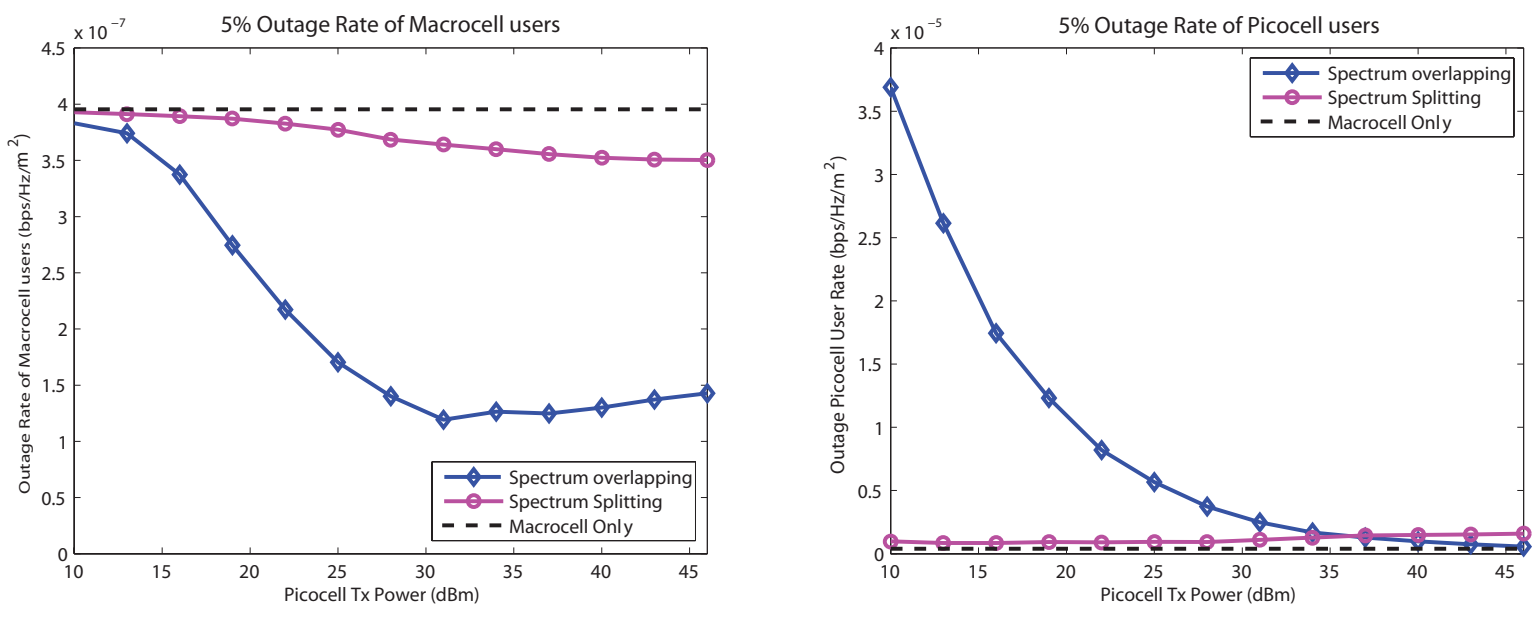

Figure 6. Outage rates of Macrocell Users and Picocell Users

the optimal picocell range was unique and could not grow too large otherwise the spectral efficiency was reduced. In our simulations, $R_{p}$ saturated around the intermediate value $400 \mathrm{~m}$ even with a picocell $\mathrm{Tx}$ power larger than the macrocell $\mathrm{Tx}$ power.

\section{CONCLUSION}

In summary, we studied the trade-off between area spectral efficiency and outage user rates in heterogeneous networks using either spectrum overlapping or spectrum splitting strategies. Although the spectrum overlapping achieved the highest spectral efficiency gain over the macrocell-only scenario, the spectrum splitting model offered a better trade-off as it could guarantee a minimum average user rate through an optimal selection of the spectrum splitting ratio and the picocell range. As an implication of this work, network operators should outweigh the goals of increasing the spectral efficiency and guaranteeing a quality service for all users. For future directions, hybrid spectrum allocation schemes may provide a better approach for managing the scarce spectrum on a large scale. In addition, the insights derived from this work clearly suggest the importance of intercell coordination and intelligent interference mitigation for protecting the macrocell users in the spectrum overlapping model. Investigation on more practical scenarios of non-symmetrically distributed cases of picocells and/or users is also considered as our future work.

\section{REFERENCES}

[1] V. Chandrasekhar, J. G. Andrews, and A. Gatherer, "Femtocell networks: a survey," IEEE Commun. Mag., vol. 46, no. 9, pp. 59-67, Sep. 2008.

[2] L.-C. Wang, G. Stuber, and C.-T. Lea, "Architecture design, frequency planning, and performance analysis for a microcell/macrocell overlaying system," IEEE Trans. Veh. Technol., vol. 46, no. 4, pp. 836-848, Nov. 1997.

[3] R. Coombs and R. Steele, "Introducing microcells into macrocellular networks: a case study," IEEE Trans. Commun., vol. 47, no. 4, pp. 568576, Apr. 1999.

[4] T. Klein and S.-J. Han, "Assignment strategies for mobile data users in hierarchical overlay networks: performance of optimal and adaptive strategies," IEEE J. Sel. Areas Commun., vol. 22, no. 5, pp. 849-861, June 2004.

[5] Motorola, "HeNB interference management," 3GPP Standard Contribution (R1-101121), San Francisco, CA, Feb. 2010.
[6] V. Chandrasekhar, J. Andrews, T. Muharemovic, Z. Shen, and A. Gatherer, "Power control in two-tier femtocell networks," IEEE Trans. Wireless Commun., vol. 8, no. 8, pp. 4316-4328, Aug. 2009.

[7] V. Chandrasekhar and J. Andrews, "Spectrum allocation in tiered cellular networks," IEEE Trans. Commun., vol. 57, no. 10, pp. 3059-3068, Oct. 2009.

[8] M. C. Erturk, H. Aki, I. Guvenc, and H, Arslan, "Fair and QoSoriented Spectrum Splitting in Macrocell-Femtocell Networks," Global Telecommunications Conference, IEEE GLOBECOM 2010, Miami, Dec. 2010.

[9] Han-Shin Jo, Ping Xia, and Jeffrey G. Andrews, "Open, Closed, and Shared Access Femtocells in the Downlink", CoRR, vol. abs/1002.2964, 2010.

[10] Madan, R.; Borran, J.; Sampath, A.; Bhushan, N.; Khandekar, A.; Tingfang Ji; , "Cell Association and Interference Coordination in Heterogeneous LTE-A Cellular Networks," Selected Areas in Communications, IEEE Journal on , vol.28, no.9, pp.1479-1489, December 2010

[11] Alouini, M.-S.; Goldsmith, A.J.; , "Area spectral efficiency of cellular mobile radio systems," Vehicular Technology, IEEE Trans. on, Jul 1999

[12] Mogensen, P.; Wei Na; Kovacs, I.Z.; Frederiksen, F.; Pokhariyal, A.; Pedersen, K.I.; Kolding, T.; Hugl, K.; Kuusela, M.; , "LTE Capacity Compared to the Shannon Bound," Vehicular Technology Conference, 2007. VTC2007-Spring. IEEE 65th , vol., no., pp.1234-1238, 22-25 April 2007

[13] P. Vieira, P. Queluz and A. Rodrigues, "Improving MIMO Spectral Efficiency in 4G Macro-Cellular Networks," Online. http://www.anacom.pt/render.jsp?categoryId=2

Table I

SiMULATION PARAMETERS

\begin{tabular}{|c|c|}
\hline Parameters & Values \\
\hline \hline System bandwidth & $9 \mathrm{MHz} / 2 \mathrm{GHz}$ \\
\hline Carrier Frequency & Macro: $46 \mathrm{dBm}$ \\
Maximum Tx Power & Pico: $10-46 \mathrm{dBm}$ \\
\hline Path loss model & $20 \log \left(\frac{4 \pi d_{0} f}{c}\right)+10 \alpha \log \left(\frac{d}{d_{0}}\right)$ \\
& $\alpha=3.76$ \\
\hline Modulation and coding & QPSK: $\frac{1}{8}, \frac{1}{5}, \frac{1}{4}, \frac{1}{3}, \frac{1}{2}, \frac{2}{3}, \frac{4}{5}$ \\
schemes & 16QAM: $\frac{1}{2}, \frac{2}{3}, \frac{4}{5}$ \\
& 64-QAM: $\frac{2}{3}, \frac{3}{4}, \frac{4}{5}$ \\
\hline Curve fitting factors for & $\eta_{W}=0.80$ \\
throughput formula in & $\eta_{S I N R}=0.91$ \\
(2) [13] & $P_{\text {error }}=10^{-3}$ \\
\hline Macrocell radius $R_{M}$ & $\frac{1}{4} \times R_{M}$ \\
\hline Macro/Pico distance & $\frac{3}{4} \times R_{M}$ \\
\hline
\end{tabular}

